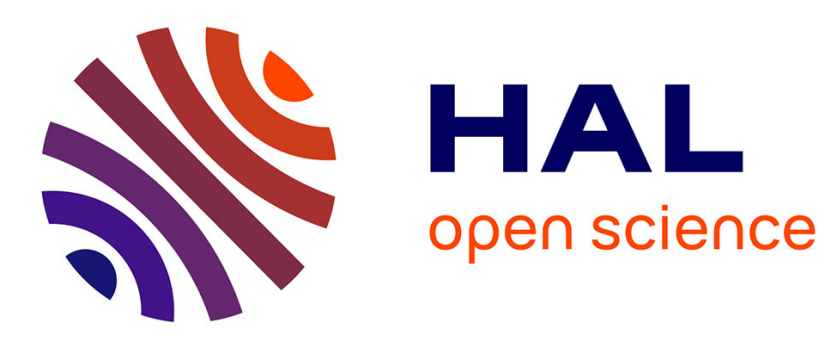

\title{
Heterogeneous multi-sensor fusion based on an evidential network for fall detection
}

\author{
Paulo Armando Cavalcante Aguilar, Jérôme Boudy, Dan Istrate, Hamid \\ Medjahed, Bernadette Dorizzi, João César Mota, Jean-Louis Baldinger, Toufik \\ Guettari, Imad Belfeki
}

\section{To cite this version:}

Paulo Armando Cavalcante Aguilar, Jérôme Boudy, Dan Istrate, Hamid Medjahed, Bernadette Dorizzi, et al.. Heterogeneous multi-sensor fusion based on an evidential network for fall detection. ICOST '11: 9th International Conference on Smart Homes and Health Telematics, Jun 2011, Montreal, Canada. pp.281-285, 10.1007/978-3-642-21535-3_42 . hal-00765028

\section{HAL Id: hal-00765028 \\ https://hal.science/hal-00765028}

Submitted on 14 Dec 2012

HAL is a multi-disciplinary open access archive for the deposit and dissemination of scientific research documents, whether they are published or not. The documents may come from teaching and research institutions in France or abroad, or from public or private research centers.
L'archive ouverte pluridisciplinaire HAL, est destinée au dépôt et à la diffusion de documents scientifiques de niveau recherche, publiés ou non, émanant des établissements d'enseignement et de recherche français ou étrangers, des laboratoires publics ou privés. 


\title{
Heterogeneous Multi-sensor Fusion Based on an Evidential Network for Fall Detection
}

\author{
Paulo Armando Cavalcante Aguilar ${ }^{1}$, Jerome Boudy ${ }^{1}$, Dan Istrate ${ }^{2}$, \\ Hamid Medjahed ${ }^{2}$, Bernadette Dorizzi ${ }^{1}$, João Cesar Moura Mota ${ }^{3}$, \\ Jean Louis Baldinger ${ }^{1}$, Toufik Guettari ${ }^{1}$, and Imad Belfeki ${ }^{1}$ \\ ${ }^{1}$ Télécom SudParis, Electronic and Physic department, Evry France \\ ${ }^{2}$ ESIGETEL, Avon, France \\ ${ }^{3}$ Federal University of Ceara, Fortaleza, Brazil \\ \{paulo. cavalcante, jerome. boudy, bernadette.dorizzi, \\ jean-louis.baldinger, toufik.guettari, \\ imad.belfeki\}@it-sudparis.eu, \\ \{dan.istrate, hamid.medjahed\}@esigetel.fr, mota@gtel.ufc.br
}

\begin{abstract}
The multi-sensor fusion can provide more accurate and reliable information compared to information from each sensor separately taken. Moreover, the data from multiple heterogeneous sensors present in the medical surveillance systems have different degrees of uncertainty. Among multi-sensor data fusion techniques, Bayesian methods and evidence theories such as Dempster-Shafer Theory (DST), are commonly used to handle the degree of uncertainty in the fusion processes. Based on a graphic representation of the DST called evidential networks, we propose a structure of heterogeneous multisensor fusion for falls detection. The proposed Evidential Network (EN) can handle the uncertainty present in a mobile and a fixed sensor-based remote monitoring systems (fall detection) by fusing them and therefore increasing the fall detection sensitivity compared to the a separated alone system.
\end{abstract}

Keywords: Remote medical monitoring, fall detection, Dempster Shafer Theory, Evidential Network.

\section{Introduction}

It is well known that multi-sensor fusion can provide more accurate and reliable information to detect distress situation for elderly persons living in their home. The data from multiple heterogeneous sensors of the medical surveillance systems present varying degrees of uncertainty and confidence [3]. Among multi-sensor fusion techniques, we can find Bayesian methods [1] and the Theory of Evidences based on the Dempster-Shafer theory (DST) [2-4, 9], which are commonly used to process and estimate degrees of uncertainty in the fusion process [4]. These theories are based on graphical representations: Bayesian Networks [1] and Evidential Networks (EN) [3,4,9].

This article investigates and implements an evidential network to detect fall situations and estimate its uncertainty degree through a heterogeneous multi-sensors fusion [3]. This network is indeed appropriate because, on one side, it prevents unreliable 
statistical model estimation due to the current lack of falls databases, and on the other side, knowing its property allowing to process heterogeneous classifiers or sensors output and to implement direct inference mechanisms on input observations such as actimetric data (body's movement, inclination) and vital data (cardiac frequency and fall index). The Evidential Networks are acyclic-directed graphs similar to Bayesian networks, but they use belief functions instead of probability functions. They are designed to handle uncertainty through the Dempster-Shafer Theory formalism.

In the following, first section 2 describes the remote medical monitoring platform constituting our targeted application, section 3 develops the Evidential Network application to the remote medical monitoring context by explaining how we implement the network and estimate the belief degree of a detected distress event such as hard or soft falls. Finally section 4 provides provisional evaluation results and section 5 concludes on this work with perspectives.

\section{Remote Medical Monitoring Platform}

A remote medical patient's monitoring system with alarm management [9], if integrated in a smart home environment, may use the result of several observation data fusion such as actimetric and vital signals captured by a device worn by the patient, external sensors such as acoustic and presence signals. Such a remote monitoring platform exists at Telecom SudParis elaborated with the close collaboration of Esigetel [8] and U558-INSERM (F. Steenkeste [6]). This Alarm management platform is composed of three detection sub-systems or modalities: GARDIEN [6, 5], RFPAT [7, 5] and ANASON [8]. In this new application work on Evidential Network, we first focused on two of these modalities: GARDIEN and RFPAT.

RFPAT system [7, 5] was designed for remote monitoring of vital and actimetric signals recorded on the person. This system can automatically identify distress situations such as falls, abrupt changes of cardiac rhythms (namely bradycardia trend) or person's activity (movements and lying down or standing posture). The GARDIEN system [6,5] consists of a network of wired or wireless infrared motion sensors placed within the smart home environment. These sensors are activated by the body movements which indicate the presence of a person in the area of interest. The person's posture inclination can also be estimated from the combination of two types of infrared sensors, one for horizontal detection field, the other vertical [6].

\section{Heterogeneous Multi-sensors Fusion for Fall Detection}

Remote monitoring systems with alarm management (RFPAT and GARDIEN) studied in this work constitute complementary information and their fusion can provide more reliable detection compared to systems used separately. RFPAT modality is already a fall detector and the purpose of this fusion is to secure the detection of falls difficult to identify such as soft falls or falls without impacts on the ground. Contextual information, such as the person's localization and interaction with the environment are very useful and should be interpreted as complementary information by the fusion process. Evidential Network is indeed appropriate to our context because one 
can avoid the problem of unreliable statistical model estimation due to the current lack of distress events data (in our case falls). Moreover Evidential Networks present a very interesting property allowing to combine heterogeneous classifiers or/and sensors processing; they also permit to implement direct inference mechanisms on input observations such as actimetric data (body's movement, inclination) and vital data (cardiac frequency and fall index).

Based on the works of Lee, Hong and Nugent [3, 4], who proposed an Evidential Network for the recognition of activities in environments such as smart homes, we propose in this work an Evidential Network for activities inference such as fall detection as shown in Figure 1. The proposed fusion approach is based on the use of the Dempster-Shafer Theory operations and rules [2-4, 9] applied to vital, actimetric and contextual information extracted from the multimodal heterogeneous alarm management system previously described in Section 2. Binary data at lower-level (IR sensors) and at higher-level (from RFPAT) constitute the input "evidences" of the considered network.

This network is structured as an acyclic graph. Hierarchy and links between nodes create dependencies between the different alarm management modalities (GARDIEN and RFPAT), which makes the fusion process more robust and reliable for abnormal

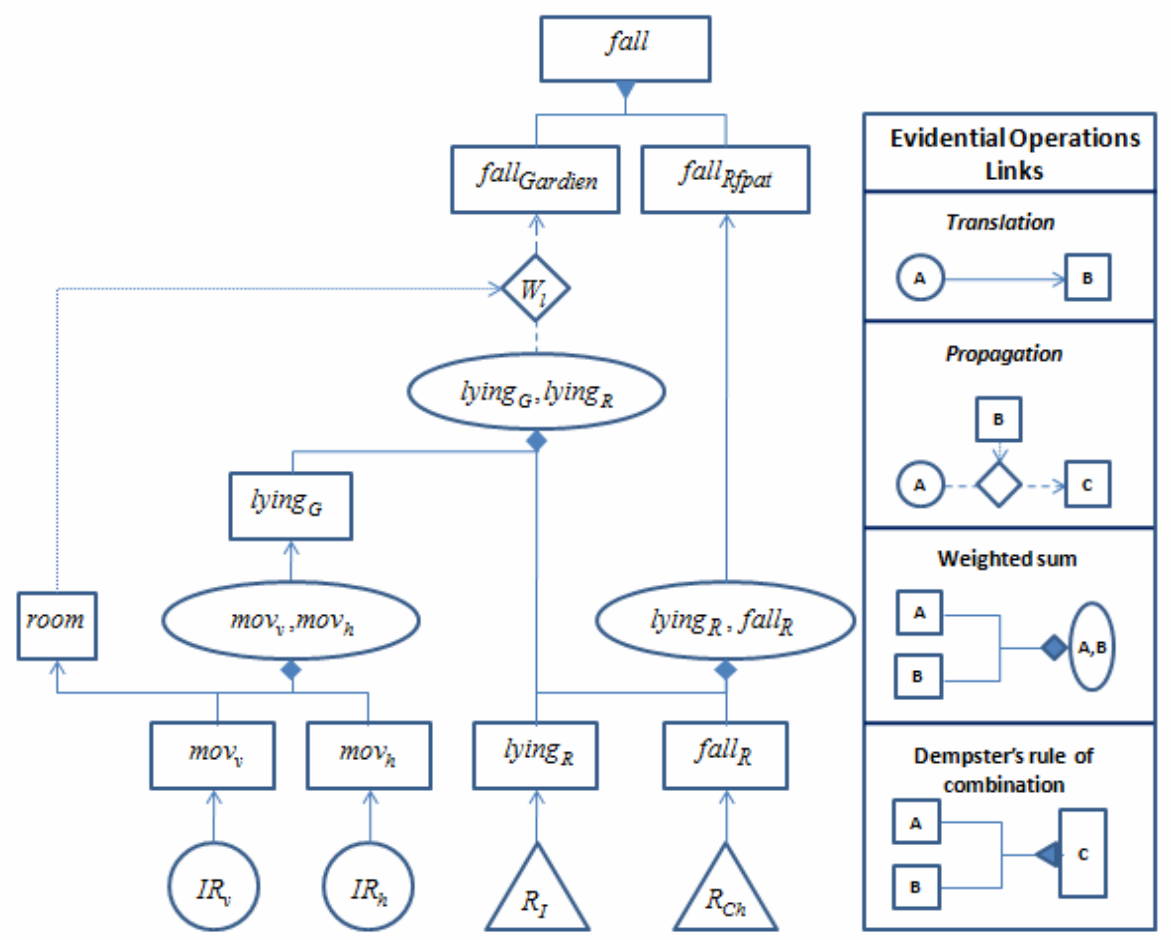

Fig. 1. Clearly the proposed network inference activities fall of person (left side). Connections between nodes represented by evidential operations (right). 
events detections. In this network (Fig. 1), the circular nodes represent the GARDIEN IR sensors: vertical " $I R_{v}$ " and horizontal " $I R_{h}$ " fields IR sensors. The triangular nodes represent the RFPAT outputs: lying down or standing posture (" $\left.R_{I} "\right)$ and fall (" $R_{C h}$ "). The square nodes represent contextual information the person's location itself ("rooms"). The rectangular nodes represent the person's activities: movement ("mov"), posture ("lying") and fall ("fall"). The connections between nodes are represented by evidential operations $[3,4,9]$, as shown in the Table on the right side of Fig. 1.

\subsection{Activity Inference of Evidential Networks}

When starting the inference process of the evidential network, we assign Belief distributions to the input nodes based on a priori sensitivity and specificity information provided by the sensors (e.g. GARDIEN) and detection devices (e.g. RFPAT) developers. Evidential operations [3, 4, 9] are used in the propagation process of inputs evidences towards the top nodes layer of the network providing the Fall decision. Each Alarm processing modality can infer a Fall status Belief, denoted $m(\{$ Chute $\})$, a Normal status Belief, $m(\{\neg$ Chute $\})$, or uncertainty degree denoted $m(\{$ Chute,$\neg$ Chute $\})$. Then the overall mass functions are fused using the combination rule of Dempster-Shafer [2-4, 9] to reach a consensus decision. Based on [3, 4], the full development of our inference process is detailed in [9].

\section{Preliminary Research Results}

The evaluation of the proposed method was performed on data recorded at Telecom SudParis. These databases consist of 5 normal and 33 fall simulated scenarios containing 16 classical falls (rather violent) and 17 soft falls (with low acceleration). The purpose of our Evidential Network-based fusion system is to detect in very specific case of soft falls.

These databases are then used to evaluate the proposed Evidential Network. To this aim a confusion matrix has been computed on normal and fall situations to evaluate the Evidential Network performance, as shown in Table 1.

Table 1. Confusion matrix of the EN fusion

\begin{tabular}{|l|l|l|l|}
\hline \multicolumn{2}{|c|}{ Confusion matrix } & \multicolumn{2}{c|}{ EN fusion } \\
\cline { 3 - 4 } & Normal & 5 & Normal \\
\hline \multirow{2}{*}{ Scenarios } & Fall & 2 & 31 \\
\cline { 2 - 4 } &
\end{tabular}

In Table 1, the EN fusion has not detected only 2 fall cases which the network is not adapted. The EN fusion presented promising good performance (sensitive of $93,94 \%$ ), in particular for soft fall cases, compared to separated modalities. The values of alone RFPAT system performance are not communicated for reasons of confidentiality due to a patent process underway. Furthermore we need to evaluate our system on a more extended database to confirm the contribution of EN fusion to the difficult falls to detect. 


\section{Conclusion and Perspectives}

Evidential Network fusion of GARDIEN and RFPAT remote monitoring modalities improved the global fall detection sensitivity with regard to separate use of the two modalities: indeed the first conducted experiments have shown good fall detection performance, which also demonstrated the interest to perform a multimodal fusion, but we still need to evaluate this approach on more extended databases reflecting more cases of users. Moreover such an evidential network is modular and can detect falls even if the RFPAT system is not present in the fusion process. For future work we propose an extension of EN to the inference of various distress situations by adding more contextual information and parameters such as pulse rate, activity, abnormal sounds, in order to better represent the occurring distress situations.

\section{Acknowledgment}

This research is funded by the FP7 European Project IST-Companion.

\section{References}

1. Becker, A., Naïm, P.: Les réseaux bayésiens. Eyrolles, eyrolles edition (1999)

2. Wu, H., Siegel, M., Stiefelhagen, R., Yang, J.: Sensor Fusion Using Dempster-Shafer Theory. In: IEEE Instrumentation and Measurement Technology Conference (May 2002)

3. Lee, H., Choi, J.S., Elmasri, R.: Sensor Data Fusion Using DSm Theory for Activity Recognition under Uncertainty in Home-Based Care. In: International Conference on Advanced Information Networking and Applications, AINA 2009 (May 2009)

4. Hong, X., Nugent, C., Mulvenna, M., McClean, S., Scotney, B., Devlin, S.: Evidential fusion of sensor data for activity recognition in smart homes. Pervasive and Mobile Computing, Pervasive Health and Wellness Management 5(3) (June 2009)

5. Medjahed, H., Istrate, D., Boudy, J., Dorizzi, B.: A Fuzzy Logic System for Home Elderly People Monitoring (EMUTEM). In: Fuzzy Systems 2009, Prague, Czech Republic (2009)

6. Steenkeste, F., Bocquet, H., Chan, M., Vellas, B.: Remote monitoring system for elders in a geriatric Hospital. In: International Conference on Aging, Arlington (December 1999)

7. Baldinger, J.L., et al.: Tele-surveillance system for patient at home: The MEDIVILLE system. In: Miesenberger, K., Klaus, J., Zagler, W.L., Burger, D., et al. (eds.) ICCHP 2004. LNCS, vol. 3118, pp. 400-407. Springer, Heidelberg (2004)

8. Istrate, D., Castelli, E., Vacher, M., Besacier, L., Serignat, J.F.: Information Extraction from Sound for Medical Telemonitoring. IEEE Transactions (April 2006)

9. Paulo, A., Cavalcante, A., et al.: Fusion multi-capteurs hétérogène basée sur un Réseau d'Evidence pour la détection de chute. In: ASSISTH Conf., Paris (January 2011) 